\title{
半导体光放大器超快相位特性的理论分析
}

\author{
陈立功, 徐天翔, 郑秀, 张尚剑, 郭小伟, 唐雄贵, 刘永* \\ 电子科技大学光电信息学院, 成都 610054 \\ * 联系人, E-mail: yongliu @uestc.edu.cn
}

2011-04-29 收稿, 2011-11-22 接受

国家重点基础研究发展计划(2011CB301705)和国家自然科学基金(60925019, 61090393, 60907008)资助

\begin{abstract}
摘要 针对目前对于半导体光放大器中的超快相位特性缺乏深入的理论分析、相关的物理 机制还不清楚的现状, 采用数值方式对半导体光放大器中的超快相位特性进行了详细的理 论分析与解释. 通过分析载流子加热、光谱烧孔等带内超快物理效应与载流子消耗等带间 效应对相位贡献的物理机制的不同, 并考虑到脉冲能量在相位响应中的作用, 研究了半导 体光放大器的相位响应特性. 在分析相位响应特性的基础上, 进一步解释了相位响应与增 益响应存在时间延迟的原因, 分析结果与已报道的实验测量结果相吻合. 理论分析结果能 够为超快光信号处理, 如光波长、全光逻辑、光波长转换、光分插复用等提供理论指导.
\end{abstract}

关键词

光信号处理 非线性光学 带内物理效应 非线性相移 半导体光放大器
半导体光放大器 (SOA)由于功耗小且具有显著 非线性效应，被广泛地应用于全光波长转换、全光逻 辑、3R 再生、全光解复用等全光信号处理 ${ }^{[1 \sim 6]}$. 但其 较慢的载流子恢复过程(典型值为几十皮秒)往往制 约着其在高速光信号处理中的应用. 目前一系列方 案已经被提出来以提高 SOA 的响应速度, 如通过增 加 SOA 的长度、增加驱动电流、采用“驻留光”(holding beam)、优化 SOA 结构参数 ${ }^{[7]}$ 等来提高 SOA 载流子 恢复速度以及采用蓝移滤波技术来消除 SOA 的慢增 益恢复过程带来的码型效应. 其中蓝移滤波技术极 大地提高了 SOA 的响应速度, 使得基于 SOA 的全光 信号处理的操作速率显著提高, 目前已经在实验中 实现了 $320 \mathrm{Gbit} / \mathrm{s}$ 无误码率的全光波长转换 ${ }^{[1]}$ 和 640 $\mathrm{Gbit} / \mathrm{s}$ 的全光解复用 ${ }^{[6]}$. 然而随着光通信、光信号处 理中使用的比特率越来越高, SOA 相位动态在光开 关、全波长转换、全光分插复用等应用中的影响将会 表现得更加明显 ${ }^{[8]}$. 其中, 世界上首次在 InP 基上制 作而成的 $8 \times 8$ 可调谐光子路由集成芯片(MOTOR)就 采用了 SOA-MZI 干涉仪结构的波长转换器, 但受限 于 SOA 带间效应导致的较慢折射率恢复时间，只能
实现单通道 $40 \mathrm{Gbit} / \mathrm{s}$ 路由速率 ${ }^{[9]}$.

目前对于 SOA 相位研究较多的是考虑带间物理 效应的影响 ${ }^{[10]}$, 而对 SOA 带内物理效应的相位响应 研究较少且存在不同观点. Giller 等人 ${ }^{[11]}$ 采用太赫兹 光学非对称解复用器(TOAD) 研究了 SOA在 $3 \mathrm{ps}$ 洜浦 脉冲作用下的相位响应, 结果表明带内物理效应几 乎不影响相位. Gomez-Iglesias 等人 ${ }^{[12]}$ 采用亚皮秒砅 浦脉冲来研究 SOA 的超快非线性效应, 实验结果中 清晰显示了带内物理效应具有明显的相位响应. 目 前对于 SOA 中产生超快相位响应的物理机制还不是 很清楚, 还缺乏明确清晰的相关理论分析. 因此分析 带内物理效应的相位响应特征以及相位响应与工作 条件的相关性是非常必要的.

光束传输法是研究无源光波导中光场传输特性 最为常用且有效的方法, 在文献[13]中光束传输方法 已成功用于研究光场在 SOA 中的传输特性. 本文中, 考虑了光谱烧孔、载流子加热、双光子吸收、自由载 流子吸收等各种超快物理效应以及增益色散和群速 色散的影响, 采用光束传输法分析了基于载流子速 率方程的光场传输特性, 建立了超快物理数值模型,

英文版见: Chen L G, Xu T X, Zheng X, et al. Theoretical analysis of ultra-fast phase recovery in semiconductor optical amplifiers. Chin Sci Bull, 2012, 57, doi: $10.1007 / \mathrm{s} 11434-011-4967-0$ 
详细分析了 SOA 相位响应动态特性.

\section{SOA 理论模型}

考虑到载流子密度变化、载流子加热以及光谱烧 孔对增益的贡献, SOA 总的增益变化可表示为

$$
g=g_{\mathrm{N}}+\Delta g_{\mathrm{CH}}+\Delta g_{\mathrm{SHB}},
$$

其中, $g_{\mathrm{N}}, \Delta g_{\mathrm{CH}}$ 和 $\Delta g_{\mathrm{SHB}}$ 分别为载流子密度、载 流子加热和光谱烧孔引起的增益的改变, 表示为

$$
\begin{aligned}
g_{\mathrm{N}} & =\alpha_{0}\left(n_{\mathrm{c}}^{*}+n_{\mathrm{v}}^{*}-n_{0}\right), \\
\Delta g_{\mathrm{CH}} & =\alpha_{0}\left(\overline{n_{\mathrm{c}}}-n_{\mathrm{c}}^{*}+\overline{n_{\mathrm{v}}}-n_{\mathrm{v}}^{*}\right), \\
\Delta g_{\mathrm{SHB}} & =\alpha_{0}\left(n_{\mathrm{c}}-\overline{n_{\mathrm{c}}}+n_{\mathrm{v}}-\overline{n_{\mathrm{v}}}\right),
\end{aligned}
$$

上式中, $\alpha_{0}$ 是增益系数, $n_{0}$ 为有源区总的态密度, $n_{x}^{*}(x \in \mathrm{c}, \mathrm{v} ; \mathrm{c}, \mathrm{v}$ 分别表示导带和价带, 下文中下标 $x$ 均表示此含义)分别为晶格温度下的导带和价带中的 载流子密度, $n_{x}$ 分别为导带和价带中的载流子密度, 导带和价带中载流子密度方程满足

$$
\begin{gathered}
\frac{\partial n_{\mathrm{c}}(z, \tau)}{\partial \tau}=\frac{\overline{n_{\mathrm{c}}}-n_{\mathrm{c}}}{\tau_{\mathrm{c}}}-v_{\mathrm{g}} g(z, \tau) S(z, \tau)-n_{\mathrm{c}} \beta_{\mathrm{c}} v_{\mathrm{g}} S(z, \tau),(5), \\
\frac{\partial n_{\mathrm{v}}(z, \tau)}{\partial \tau}=\frac{\overline{n_{\mathrm{v}}}-n_{\mathrm{v}}}{\tau_{\mathrm{v}}}-v_{\mathrm{g}} g(z, \tau) S(z, \tau)-n_{\mathrm{v}} \beta_{\mathrm{v}} v_{\mathrm{g}} S(z, \tau),
\end{gathered}
$$

式中, $\overline{n_{x}}$ 分别为准费米分布下的导带和价带中的载 流子密度, $\tau_{x}$ 分别为导带和价带中的载流子-载流子 散射时间, $\beta_{x}$ 分别为导带和价带中的自由载流子吸 收系数, $s(z, \tau)$ 是光场强度.

对于式(1)中的 SOA 增益, 在脉冲较宽、增益带 宽较小时, 可近似认为其在光谱范围内是不变的. 随 着脉冲宽度降低, 脉冲的光谱谱宽展宽, 当脉冲宽度 小于 $10 \mathrm{ps}$ 时, SOA 的增益在脉冲光谱范围内不能再 视为是均匀的, 必须考虑增益随频率的变化 ${ }^{[14]}$, 因 此在分析短脉冲作用下的 SOA 响应特性时必须考虑 增益色散. 同时由于 SOA 相位变化与增益变化是由 线宽增强因子建立联系的, 所以群速色散必然伴随 着增益色散出现, 因此也必须考虑群速色散的影响. 在这里我们假设 SOA 的增益谱为抛物线型, 可以将 式(1)展开为泰勒级数

$$
g(\tau, \omega)=g\left(\tau, \omega_{0}\right)+\left.\Delta \omega \frac{\partial g(\tau, \omega)}{\partial \omega}\right|_{\omega_{0}}+\left.\frac{\Delta \omega^{2}}{2} \frac{\partial^{2} g(\tau, \omega)}{\partial \omega^{2}}\right|_{\omega_{0}},
$$

式中, 对于一阶微分 $\left.\frac{\partial g(\tau, \omega)}{\partial \omega}\right|_{\omega_{0}}$ 和二阶微分

$$
\begin{aligned}
\left.\frac{\partial^{2} g(\tau, \omega)}{\partial \omega^{2}}\right|_{\omega_{0}} \text { 可进一步简化为 }{ }^{[15]} & \\
& \left.\frac{\partial g(\tau, \omega)}{\partial \omega}\right|_{\omega_{0}}=A_{1}+B_{1}\left[g_{0}-g(\tau, \omega)\right], \\
& \left.\frac{\partial^{2} g(\tau, \omega)}{\partial \omega^{2}}\right|_{\omega_{0}}=A_{2}+B_{2}\left[g_{0}-g(\tau, \omega)\right],
\end{aligned}
$$

其中 $A_{1}, A_{2}$ 分别为在频率 $\omega_{0}$ 处线性增益曲线的斜率; $B_{1}, B_{2}$ 为饱和效应引起的增益改变率.

对于 SOA 的相位 $\Phi$, 我们采用 Kramers-Kronig 关系来建立与增益的联系. 通过线宽提升因子 $\alpha$, 相位在 SOA 中的变化可表示为

$$
\frac{\partial \Phi}{\partial z}=-\frac{1}{2} \alpha g .
$$

综合以上分析, 并考虑双光子效应的影响, 光场 传输方程可表示为

$$
\begin{aligned}
\frac{\partial A(z, \tau)}{\partial z}= & \left\{\frac{1}{2} \Gamma g_{0}(z, \tau)-\left.\Gamma \frac{i}{2} \frac{\partial g(\tau, \omega)}{\partial \omega}\right|_{\omega_{0}} \frac{\partial}{\partial \tau}\right. \\
& -\left.\frac{1}{4} \Gamma \frac{\partial^{2} g(\tau, \omega)}{\partial^{2} \omega}\right|_{\omega_{0}} \frac{\partial^{2}}{\partial^{2} \tau} \\
& -\frac{1}{2} \Gamma_{2} \beta_{2}\left(1+i \alpha_{2}\right)|A(z, \tau)|^{2} \\
& -\frac{1}{2} \Gamma \beta_{\mathrm{c}} n_{\mathrm{c}}(z, \tau)-\frac{1}{2} \Gamma \beta_{\mathrm{v}} n_{\mathrm{v}}(z, \tau) \\
& \left.+\frac{1}{2} \beta_{\mathrm{GVD}} \frac{\partial^{2}}{\partial^{2} \tau}-\frac{1}{2} \alpha_{\mathrm{int}}+i \Phi(z, \tau)\right\} A(z, \tau),
\end{aligned}
$$

式中, $\Gamma$ 为限制因子, $\beta_{2}$ 是双光子吸收系数, $\beta_{\mathrm{GVD}}$ 为群速色散系数, $\alpha_{\mathrm{int}}$ 是内部损耗系数.

\section{2 数值模拟结果与分析}

在数值计算中, SOA 被分为 60 段, 每段 SOA 内 的物理参量均视为常量. 对于(11)式采用有限差分光 束传播的方法 (FD-BPM) 来求解方程. 在计算中, SOA 有源区长度为 $300 \mu \mathrm{m}$, 有源区的体积为 $60 \mu \mathrm{m}^{3}$, SOA 注人电流为 $210 \mathrm{~mA}$, 输人洜浦脉冲为一阶高斯 脉冲, 探测光为直流光. 其余数值模拟中使用物理参 数如表 1 所示, 其中所使用主要参数均为相关文献中 使用的参数值 ${ }^{[9,13,16]}$.

图 1 是 $2 \mathrm{ps}$ 的葲浦光人射下的 SOA 归一化增益, 其中实线部分为实验结果, 虚线部分为采用上述 数值模型的计算结果. 从图中可以看出此模型能 够准确地描述 SOA 带内以及带间物理效应的载流 子动态. 
表 1 SOA 的参数定义及数值

\begin{tabular}{ccc}
\hline 参数 & 符号 & 参数值 \\
\hline 总的态密度 & $N_{0}$ & $3.244 \times 10^{5} \mu \mathrm{m}^{-3}$ \\
群速色散系数 & $\beta_{\mathrm{GVD}}$ & $5 \times 10^{6} \mathrm{ps}^{2} / \mathrm{mm}$ \\
载流子寿命 & $\tau_{\mathrm{s}}$ & $100 \mathrm{ps}$ \\
导带中载流子-载流子散射时间 & $\tau_{\mathrm{c}}$ & $0.1 \mathrm{ps}$ \\
价带中载流子-载流子散射时间 & $\tau_{\mathrm{v}}$ & $0.05 \mathrm{ps}$ \\
导带中载流子-声子散射时间 & $\tau_{\mathrm{CR}}$ & $0.75 \mathrm{ps}$ \\
价带中载流子-声子散射时间 & $\tau_{\mathrm{VR}}$ & $0.25 \mathrm{ps}$ \\
自由载流子吸收系数 & $\beta_{\mathrm{c}}, \beta_{\mathrm{v}}$ & $1 \times 10^{-9} \mu \mathrm{m}^{-1}, 0 \mu \mathrm{m}^{-1}$ \\
双光子吸收系数 & $\beta_{2}$ & $3.6 \times 10^{-7} \mu \mathrm{m}^{2}$ \\
波导损耗系数 & $\alpha_{\mathrm{int}}$ & $1.75 \times 10^{-3} \mu \mathrm{m}^{-1}$ \\
模场限制因子 & $\Gamma, \Gamma_{2}$ & $0.28,0.5$ \\
群速度 & $\nu_{\mathrm{g}}$ & $100 \mu \mathrm{m} / \mathrm{ps}$ \\
线宽提升因子 & $\alpha_{\mathrm{N}}$ & 7 \\
线宽提升因子 & $\alpha_{\mathrm{CH}}$ & 3 \\
线宽提升因子 & $\alpha_{\mathrm{SHB}}$ & 0.1 \\
\hline
\end{tabular}

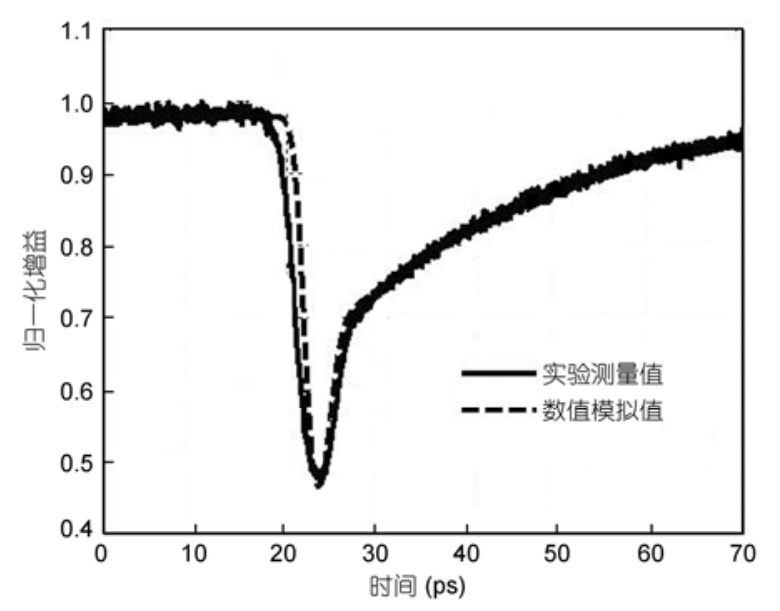

图 1 不浦脉冲宽度为 2 ps 时的增益动态 虚线为数值模拟结果, 实线为实验结果

图 2 是 $18.1 \mathrm{~mW}$ 峰值功率下的不同脉冲宽度的 泵浦脉冲作用下 SOA 的相位响应. 从图中可以看到, 当泵浦脉冲宽度为 $3 \mathrm{ps}$ 时, 相位恢复主要由较慢恢 复过程主导, 此结果与 Giller 等人 ${ }^{[11]}$ 的实验结果吻合. 随着脉冲宽度的降低, 超快相位恢复过程逐渐凸显 出来. 当脉冲宽度为 $1 \mathrm{ps}$ 时, SOA 相位恢复显示出明 显的超快恢复过程, 此时结果与 Gomez-Iglesias 等

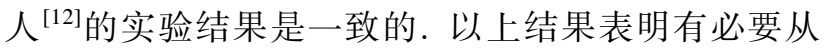
原理上来进一步分析形成此结果的原因.

图 3 分别是相同峰值功率下的导带和价带的载 流子温度动态. 从图中可以看出, 导带中的电子和价 带中空穴的最高温度分别约为 322 和 $300.7 \mathrm{~K}$, 载流

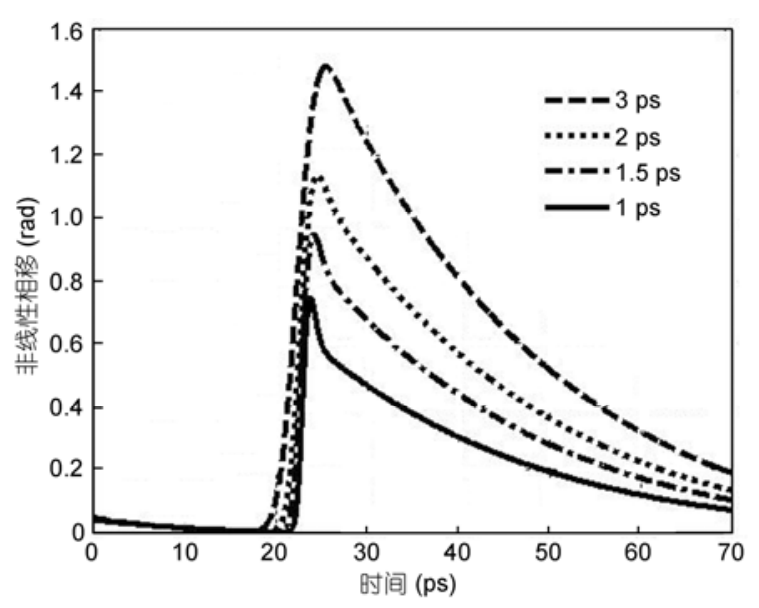

图 2 峰值功率 $18.1 \mathrm{~mW}$, 脉冲宽度分别为 $1,1.5,2$ 和 $3 \mathrm{ps}$ 的泵浦脉冲作用下的相位响应曲线
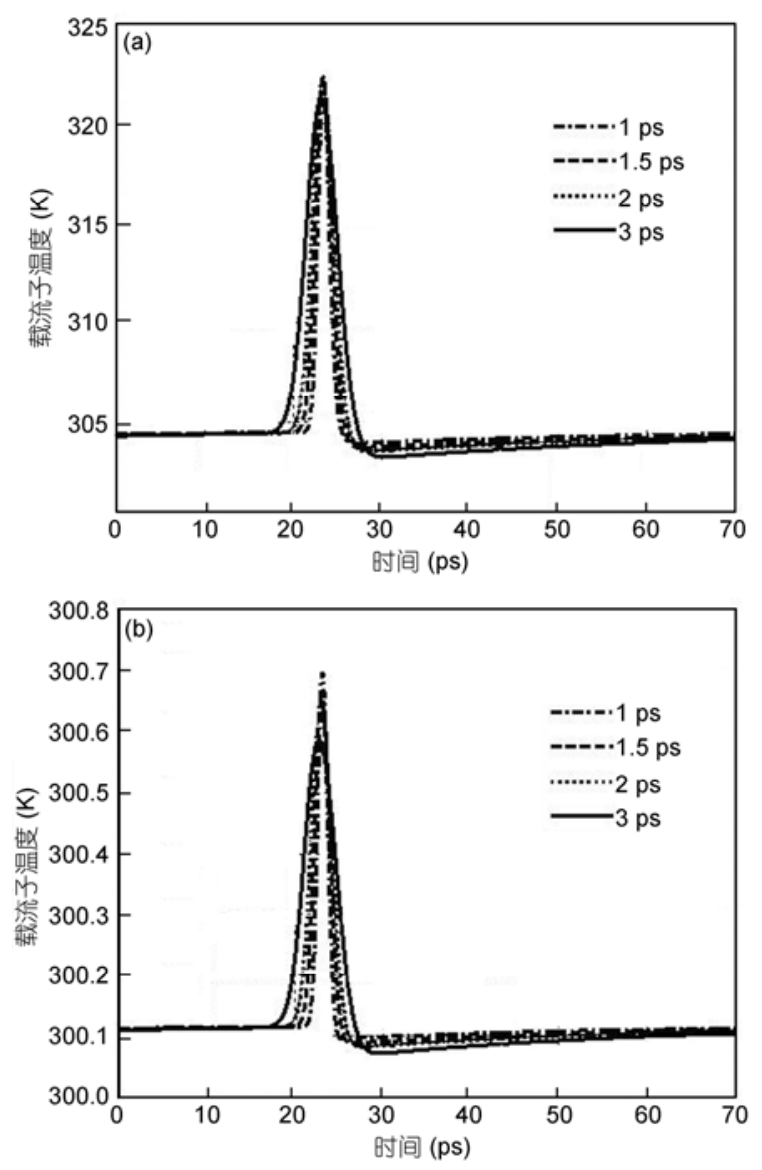

图 3 峰值功率 $18.1 \mathrm{~mW}$, 脉冲宽度分别为 $1,1.5,2$ 和 $3 \mathrm{ps}$ 的洜浦脉冲作用下, 半导体光放大器导带 (a) 和价带 (b) 中的 载流子温度曲线

子加热效应主要由导带中的电子贡献, 而空穴对载 流子加热效应几乎没有贡献, 这是空穴相对于电子 
具有更大的有效质量、空穴温度弛豫时间较短引起的. 由于载流子温度的升高, 导带和价带中的载流子将 形成新的热平衡分布. 载流子从热平衡分布到准费 米分布过程中由于温度的升高使得导带底的载流子 数目减少, 然后由于流子-声子散射, 温度迅速驰豫 到晶格温度, 载流子密度逐渐恢复, 这一过程中载流 子-声子散射时间约为 $1 \mathrm{ps}$, 因此载流子加热能够引 起超快的增益恢复. 载流子加热对于 SOA 的相位响 应的影响与其对增益的影响不同. 载流子加热效应 使得导带底的载流子跃迁到高的能级这一过程中, 载流子密度分布的变化对相位影响较小, 因此在数 值模拟中采用了一个小的 $\alpha_{\mathrm{CH}}$.

在图 3 中, 对比不同脉宽的温度动态, 可以发现 在相同峰值功率下, 导带和价带载流子的最高温度, 并没有发生明显变化, 也就是说对于不同的脉冲宽 度载流子加热效果并没有明显的区别, 可见超快相 位恢复过程并不是完全由载流子加热过程决定的. 为了解释这一现象, 需要分析原浦光人射时 SOA 中 所产生各种物理机制之间的关系.

图 4 是 $2 \mathrm{ps}$ 泵浦脉冲作用下分别由载流子加 热、光谱烧孔和载流子密度变化引起的增益变化 $g_{\mathrm{N}}$, $\Delta g_{\mathrm{CH}}, \Delta g_{\mathrm{SHB}}$. 随着百浦脉冲的人射, 光谱烧孔和载 流子加热效应几乎同时发生, 引起增益变化 $\Delta g_{\mathrm{CH}}$, $\Delta g_{\mathrm{SHB}}$, 随即热的载流子经由载流子-声子散射而弛豫 到晶格温度, 而受激发射引起的光谱“谱洞”也由 带内流子-载流子散射而迅速被填平, 产生了超快的 增益恢复. 载流子密度变化则表现出与上述超快

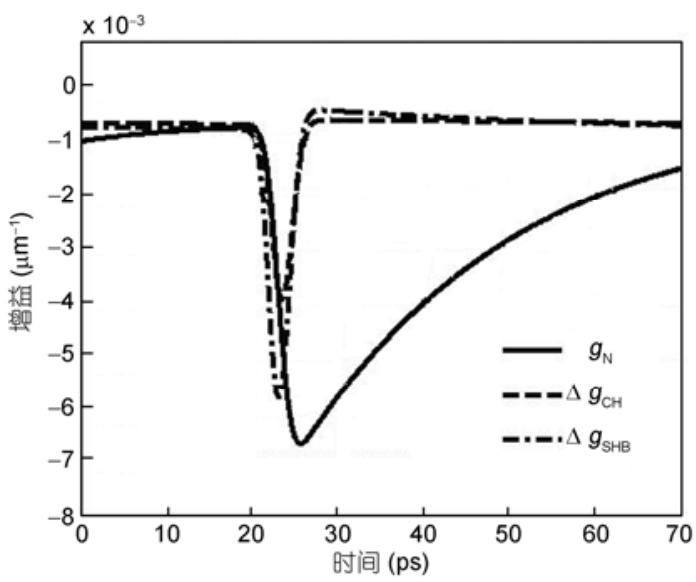

图 42 ps 脉冲宽度为下的归一化增益响应

其中 $\Delta g_{\mathrm{CH}}, \Delta g_{\mathrm{SHB}}, g_{\mathrm{N}}$ 分别为与载流子加热、光谱烧孔和载流子密度 变化相关联的增益变化
物理效应不同的特性, 从图中可以看出载流子密度 则是在载流子加热以及光谱烧孔过程即将结束的时 刻降低到最小值, 这主要是由于载流子密度变化是 与进人 $\mathrm{SOA}$ 光场强度和注人电流决定的. 由于载流 子密度变化与进人 SOA 有源区的光场强度密切相关, 因此带间物理过程相对于带内物理过程的滞后时间 是与脉冲宽度联系在一起的. 当载流子密度达到最 小时, 受激发射引起的载流子耗竭与载流子注人率 之间达到平衡状态. 此后载流子密度变化则主要由 电流的注人率决定, 增益动态进人慢的恢复过程. 在 葲浦脉冲人射情况下，在不同时刻，脉冲响应由不同 物理机制所主导.

超快的带内物理效应和载流子密度变化的物理 过程的不同步决定超快相位响应的程度. 在脉冲宽 度较宽时 (大于 $3 \mathrm{ps}$ ), 由于带内物理机制的物理过程 和带间物理机制的物理过程的时间延迟较大, 因此 超快的相位响应并不明显. 随着脉冲宽度的降低, 带 间效应的作用过程更加趋于与温度弛豫过程一致, 因此超快的相位响应更加明显. 此外, 随着脉冲宽度 的增加, 脉冲能量增大, 载流子密度变化在相位响应 中的贡献也随之增大, 也使得相位的超快恢复过程 产生相应缩减.

带内物理机制和带间物理机制脉冲响应特性的 不同，也导致了相位响应和增益响应存在不同的表 现. 图 5(a)是 $2 \mathrm{ps}$ 原浦脉冲人射下, SOA 的增益动态 和相位动态, 对比相位曲线和增益曲线可以看出相 位峰值滞后于增益峰值, 这一结论与文献 $[17,18]$ 中 的观察结果是一致的. 图 5(b) 是不同脉冲宽度下的延 迟时间，显示了延迟时间与脉冲宽度紧密相关. 这一 结果的原因是相位响应主要是由载流子密度决定, 带内物理效应贡献较小; 而在增益响应中超快的增 益动态主要由内带物理效应决定，所以不同物理机 制在不同的时刻分别主导相位和增益，因而产生了 时延. 同时由于载流子密度变化与有源区的脉冲能 量相关，时延不仅与脉冲宽度相关，同时也随着能量 的增加有细微的增大.

\section{3 结论}

本文数值分析了载流子加热、光谱烧孔以及载流 子耗竭等各种超快物理效应在 SOA 相位响应中的影 响. 结果显示由于载流子消耗与载流子加热以及光 谱烧孔等带内物理效应分别在脉冲响应过程中的不 

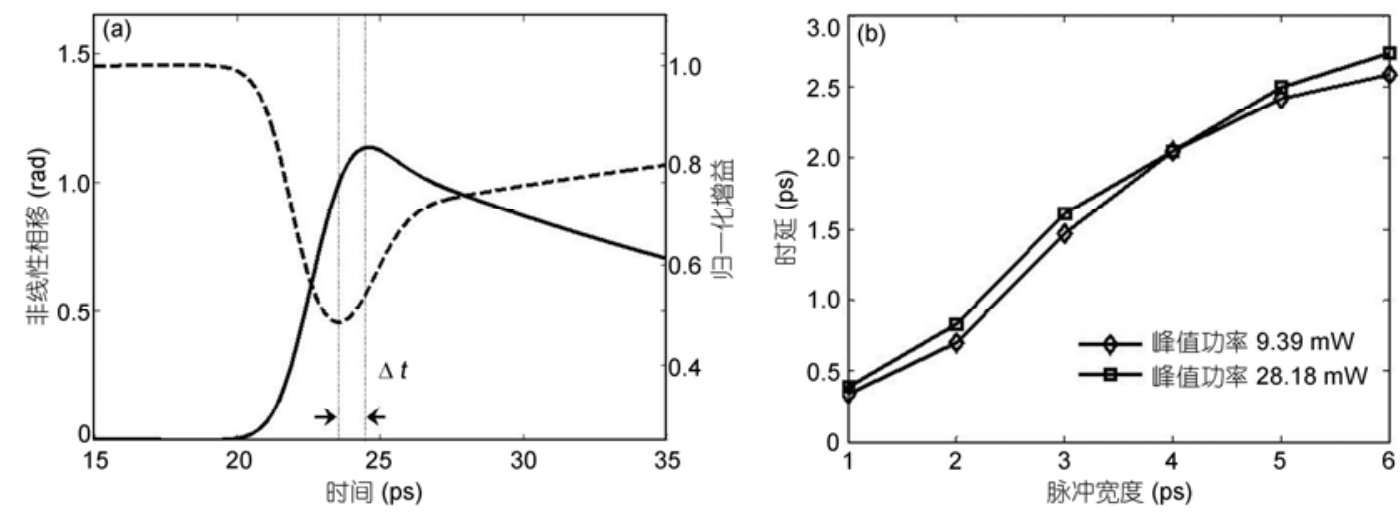

图 5 (a) $2 \mathrm{ps}$ 脉冲宽度下的相位响应与增益响应的时延; (b) 时延分别在脉冲峰值功率 9.39 和 $28.18 \mathrm{~mW}$ 下随脉冲 宽度的变化

同时刻处于主导地位，使得超快的相位动态不仅与 带内物理效应相关, 也与脉冲宽度紧密相关. 当脉冲 较宽时, 由于带间物理过程滞后于载流子温度弛豫 过程, 相位响应主要由载流子密度决定, 因此没有明 显的超快响应. 随着脉冲宽度的降低, 带间物理过程 趋向于与温度弛豫过程一致, 因此产生超快相位响
应. 同时由于带内物理效应在增益响应处于主导地 位, 而 SOA 相位响应则主要受载流子密度变化影响, $\mathrm{SOA}$ 的相位动态相对于增益动态存在着时间延迟. 以上对于 SOA 相位特性的分析结果将能够应用于基 于 SOA 的超高速的全关信号处理, 解决 SOA 相位动 态对光开关、光波长转换以及光分插复用的影响.

\section{参考文献}

1 Liu Y, Tangdiongga E, Li Z, et al. Error-free $320 \mathrm{~Gb} / \mathrm{s}$ all-optical wavelength conversion using a single semiconductor optical amplifier. J Lightwave Technol, 2007, 25: 103-108

2 Ni Y L, Wang Z Y. Experimental analysis of all optical wavelength conversion using XGM in SOA (in Chinese). Chin Sci Bull, 2009, 54: 3024-3029

3 Zhang X, Wang Y, Sun J, et al. All-optical AND gate at $10 \mathrm{Gbit} / \mathrm{s}$ based on cascaded single-port-couple SOAs. Opt Express, 2004, 12: 361-366

4 Dong J, Zhang X, Fu S, et al. Ultra fast all-optical signal processing based on single semiconductor optical amplifier and optical filtering. IEEE J Sel Top Quant, 2008, 14: 770-778

5 Duan P X, Chen L G, Zhang S J, et al. All-optical 2R regeneration based on self-induced polarization rotation in a single semiconductor optical amplifier. Chin Sci Bull, 2009, 54: 3704-3708

6 Tangdiongga E, Liu Y, De W H, et al. All-optical demultiplexing of 640-to-40-Gb/s using filtered chirp of a semiconductor optical amplifier. Opt Lett, 2007, 32: 835-837

7 Huang X, Qin C, Huang D, et al. Local carrier recovery acceleration in quantum well semiconductor optical amplifiers. IEEE J Quantum Electron, 2010, 46: 1407-1413

8 Nielsen M L, Mørk J. Experimental and theoretical investigation of the impact of ultra-fast carrier dynamics on high speed SOA-based all-optical switches. Opt Express, 2006, 14: 331-347

9 Nicholes S C, Masanovic M L, Jevremovic B, et al. An $8 \times 8$ InP monolithic tunable optical router (MOTOR) packet forwarding chip. J Lightwave Technol, 2010, 28: 641-650

10 Schares L, Schubert C, Schmidt C, et al. Phase dynamics of semiconductor optical amplifiers at 10-40 GHz. IEEE J Quantum Electron, 2003, 39: 1394-1408

11 Giller R, Manning R J, Cotter D. Gain and phase recovery of optically excited semiconductor optical amplifiers. IEEE Photonic Tech L, 2006, 18: 1061-1063

12 Gomez-Iglesias A, Fenn J G, Mazilu M, et al. Carrier heating in semiconductor optical amplifier-based sagnac-type all-optical switches. Semicond Sci Tech, 2006, 21: 1703-1708 
13 Hong M Y, Chang Y H, Dienes A, et al. Femtosecond self- and cross-phase modulation in semiconductor laser amplifiers. IEEE J Sel Top Quant, 1996, 2: 523-539

14 Agrawal G P. Effect of gain dispersion on ultrashort pulse amplification in semiconductor laser amplifiers. IEEE J Quantum Electron, 1991, 2: 1843-1849

15 Dienes A, Heritage J P, Hong M Y, et al. Time- and spectral-domain evolution of sub-picosecond pulses in semiconductor optical amplifiers. Opt Lett, 1992, 17: 1602-1604

16 Mark J, Mørk J. Subpicosecond gain dynamics in InGaAsP optical amplifiers: Experiment and theory. Appl Phys Lett, 1992, 61: 22812283

17 Wang J, Maitra A, Poulton C G, et al. Temporal dynamics of the alpha factor in semiconductor optical amplifiers. J Lightwave Technol, 2007, 25: 891-900

18 Dailey J M, Koch T L. Impact of carrier heating on SOA dynamics for wavelength conversion. In: Proceeding of Lasers and Electro-Optical Society. Montreal, Qua, IEEE, 2006. 156-157 